\title{
Manifolds on the verge of a hyperbolicity breakdown
}

\author{
À. Haro \\ Departament de Matemàtica Aplicada i Anàlisi, Universitat de Barcelona, 08007 Barcelona, Spain \\ R. de la Llave \\ Department of Mathematics, University of Texas, Austin, Texas 78712 and Institució Catalana de Recerca $i$ \\ Estudis Avançats, Lluís Companys 23, 08010 Barcelona, Spain
}

(Received 5 September 2005; accepted 21 November 2005; published online 8 March 2006)

\begin{abstract}
We study numerically the disappearance of normally hyperbolic invariant tori in quasiperiodic systems and identify a scenario for their breakdown. In this scenario, the breakdown happens because two invariant directions of the transversal dynamics come close to each other, losing their regularity. On the other hand, the Lyapunov multipliers associated with the invariant directions remain more or less constant. We identify notable quantitative regularities in this scenario, namely that the minimum angle between the two invariant directions and the Lyapunov multipliers have power law dependence with the parameters. The exponents of the power laws seem to be universal. (C) 2006 American Institute of Physics. [DOI: 10.1063/1.2150947]
\end{abstract}

\begin{abstract}
Quasiperiodically forced systems occur in many situations in physics, mathematics, engineering, etc. In many cases, the external quasiperiodic perturbations induce quasiperiodic motions, which correspond to invariant tori. It is important to understand when these invariant tori persist under perturbations, and to identify the mechanisms of their breakdown. It has been known for a long time that the persistence of a torus is related to the exponential growth of the linearization along certain directions (normal hyperbolicity), and that normal hyperbolicity may be lost because of bifurcations such as saddle-node and period doubling, among others. The common feature of these standard bifurcations is that some Lyapunov multipliers approach 1, while the invariant directions remain smooth. In this paper we propose a new mechanism, in which two invariant directions of the linearized dynamics come close to each other, losing their regularity, and the corresponding Lyapunov multipliers remain more or less constant, away from each other and away from 1. Hence, the phenomenon is described by two observables: the minimum angle between the invariant directions and the Lyapunov multipliers. We also identify notable universal power laws of these observables.
\end{abstract}

\section{INTRODUCTION}

The long-term behavior of a dynamical system is organized by its invariant objects. Hence, it is important to understand which invariant objects persist under perturbations of the system. It has been known for a long time that the persistence of an invariant object is related to the exponential rate of growth of the perturbations of orbits starting on it.

For example Refs. 1-3 show that a manifold persists under all $C^{1}$ small changes in the map if it is normally hyperbolic. The fact that this condition is also necessary for $C^{1}$ persistence was proven in Ref. 4.

A problem that has received a great deal of attention ${ }^{5-7}$ is the study of the breakdown of normally hyperbolic invariant manifolds.
We recall that a manifold is normally hyperbolic if all the perturbations transversal to the manifold grow at an exponential rate (either in the future or in the past), and that this exponential rate is bigger than the exponential rate of growth for perturbations tangential to the manifold. We will not give a precise definition of normal hyperbolicity in general, but we refer to the literature quoted above. In Eq. (4) we will give the definition of a more general concept (exponential dichotomy) tailored for the systems that we will consider in this paper, which are quasiperiodically forced maps [see Eq. (1)].

We note that the definition of hyperbolicity has two measures of quality. One is the asymptotic rate of growth [called $\lambda_{ \pm}$in Eq. (4)] and another is the prefactor in the exponential [called $C$ in Eq. (4)], which measures how long it is necessary to wait to observe the asymptotic rate of growth.

Even if most rigorous studies of loss of hyperbolicity are concerned with situations in which the rates of growth $\lambda_{ \pm}$ degenerate, we emphasize (see also Ref. 8) that hyperbolicity may well be lost because the prefactors $C$ become unbounded.

Indeed, in this paper we report two situations in which the hyperbolicity (or more generally the exponential dichotomy) is lost because the prefactors grow unbounded even though the asymptotic exponential rates remain more or less constant. In other words, even though the asymptotic rates of growth remain more or less constant we have to observe them for increasingly long time intervals till they manifest themselves.

Interestingly, we find in the situations above that there are quantitative regularities and scaling behaviors for several observables. The scaling exponents seem to be universal in a wide class of systems.

In this paper, we find invariant tori and study in detail the dynamics of the linearized equations around them [see Eq. (3)]. In particular, we compute quite accurately the invariant directions and their corresponding Lyapunov multi- 
pliers [see Eq. (5)]. One of the techniques we use is the reduction of the linearized system to a constant matrix. The theory in Ref. 9 allows us to assess the reliability of the calculations.

The most interesting finding of our studies is that, for some systems at the boundary of hyperbolicity, there are scalings in the asymptotic behavior of several observables, and that the scaling exponents do not seem to depend on the system. See Assertion 1 for a detailed formulation.

At the moment, we do not have a rigorous explanation of this universal scaling behavior. It is natural to conjecture that there should be a renormalization group formulation, but we have also observed that the scaling does not seem to depend on the frequency of the forcing as is the case with most renormalization group formulations hitherto found in the literature.

Though we do not have an explanation, we present an alternative description of the problem in terms of spectral properties of some linear operators associated naturally to the invariant tori, called transfer operators [see Eq. (10)]. The phenomenon we have found is closely related to the sudden growth of the spectrum of those transfer operators.

The phenomenon observed can also be described in more geometric terms. It is well known that to a hyperbolic system (more generally, to a system with an exponential dichotomy) one can associate invariant directions corresponding to vectors with different asymptotic rates of growth. The phenomenon we have found corresponds to the fact that these directions get closer and that their distance satisfies certain scaling relations regardless of the family of maps considered.

To understand geometrically the phenomenon of merging of the invariant directions, it is natural to look at the "projectivized dynamics," that is, the dynamics in the directions of the vectors transversal to the torus. We find empirically that the phenomenon observed is visually very similar to the collision mechanism for the formation of strange nonchaotic attractors described in Refs. 6 and 10-13. We present a detailed study in Sec. V.

\section{SETUP OF THE PROBLEM}

We study smooth dynamical systems of the form

$$
\bar{x}=f(x, \theta), \quad \bar{\theta}=\theta+\omega,
$$

where $x \in \mathbb{R}^{n}, \theta \in \mathbb{T}^{d}$ [defined $\left.(\bmod 1)\right]$, and $\omega \in \mathbb{R}^{d}$ is fixed. This class of dynamical systems models physical systems subject to external quasiperiodic perturbations, of frequency $\omega$. In this paper, we will report only numerical experiments for $n=2, d=1, \omega$ irrational, and we will restrict the discussion to this case for the sake of simplicity. The general theory in Refs. 9 and 14 works for arbitrary $n, d$.

A natural way ${ }^{9}$ to study invariant tori $\mathcal{K}$ for Eq. (1) is to find a continuous map $K: \mathrm{T} \rightarrow \mathbb{R}^{2}$ in such a way that

$$
f(K(\theta), \theta)=K(\theta+\omega) .
$$

Equation (2) shows that a point $\mathcal{K}(\theta)=(K(\theta), \theta)$ is mapped onto another point of the same form. Once a torus $\mathcal{K}$ has been located, it is natural to consider the linearization around it. The linearized dynamics is given by the cocycle $M$

$$
\bar{v}=M(\theta) v, \quad \bar{\theta}=\theta+\omega,
$$

where $M(\theta)=D_{x} f(K(\theta), \theta)$. We can think of the vectors $v$ as small perturbations transversal to the torus, and Eq. (3) describes their growth under iteration. (Notice that small perturbations tangential to the torus do not grow because the dynamics on the torus is a rigid rotation.)

We denote by $M^{m}(\theta)=M(\theta+(m-1) \omega) \cdots M(\theta)$ the result of advancing the linearized dynamics $m$ units of time; $M^{-m}(\theta)=M(\theta-m \omega)^{-1} \cdots M(\theta-\omega)^{-1}$ denotes the result of moving back the linearized dynamics $m$ units of time.

We say that the invariant torus has a spectral gap (or that it has an exponential dichotomy, following Ref. 15) when we can find numbers $C>0,0<\lambda_{-}<\lambda_{+}$, and a splitting $\mathbb{R}^{2}=E_{\theta}^{-} \oplus E_{\theta}^{+}$in $1 \mathrm{D}$ subspaces $E_{\theta}^{ \pm}$characterized by

$$
\begin{aligned}
& v \in E_{\theta}^{-} \Leftrightarrow\left|M^{+m}(\theta) v\right| \leqslant C\left(\lambda_{-}\right)^{+m}|v|, \quad m \geqslant 0, \\
& v \in E_{\theta}^{+} \Leftrightarrow\left|M^{-m}(\theta) v\right| \leqslant C\left(\lambda_{+}\right)^{-m}|v|, \quad m \geqslant 0 .
\end{aligned}
$$

Note that the definition implies that $E_{\theta}^{ \pm}$are invariant. That is, $M(\theta) E_{\theta}^{ \pm}=E_{\theta+\omega}^{ \pm}$. Informally, $E_{\theta}^{+}$consists of the vectors based at $\mathcal{K}(\theta)$ that grow at an exponential rate bigger than $\lambda_{+}$in the future and those in $E_{\theta}^{-}$grow at a rate smaller than $\lambda_{-}$in the future. Moreover, the splitting $\mathbb{R}^{2}=E_{\theta}^{-} \oplus E_{\theta}^{+}$defines two projections $P_{\theta}^{ \pm}$, over the spaces $E_{\theta}^{ \pm}$.

Notice also that with fixed $\theta \in \mathbb{T}$, the directions of the future iterates $M^{m}(\theta) v$ of a vector $v \notin E_{\theta}^{-}$will tend to the directions of $E_{\theta+m \omega}^{+}$. For this reason, we will refer to $E_{\theta}^{+}$as the attracting direction, and to $E_{\theta}^{-}$as the repelling direction.

When $\lambda_{-}<1<\lambda_{+}$the torus is normally hyperbolic, of saddle type, and $E_{\theta}^{ \pm}$are the unstable $(+)$and stable (-) directions. If $\lambda_{-}<\lambda_{+}<1$, the torus is also normally hyperbolic, of attracting-node type, and $E_{\theta}^{ \pm}$are the slow (+) and fast (-) stable directions.

The invariant directions $E_{\theta}^{ \pm}$are also characterized by the Lyapunov multipliers. These are the asymptotic rates of growth $\Lambda^{ \pm}$given by

$$
\begin{aligned}
& \Lambda^{+}=\lim _{m \rightarrow \pm \infty}\left|M^{m}(\theta) v\right|^{1 / m}, \quad v \in E_{\theta}^{+} \backslash\{0\}, \\
& \Lambda^{-}=\lim _{m \rightarrow \pm \infty}\left|M^{m}(\theta) v\right|^{1 / m}, \quad v \in E_{\theta}^{-} \backslash\{0\} .
\end{aligned}
$$

In our case, if the invariant torus is normally hyperbolic, then the fact that the dynamics on the torus is a rotation implies that the torus is smooth ${ }^{9}$ and its invariant directions $E_{\theta}^{ \pm}$depend smoothly on $\theta \cdot{ }^{9,16,17}$ Furthermore, using the facts that irrational rotations are uniquely ergodic and the directions are one-dimensional, it is shown in Ref. 18 that if the bundles are continuous the limits in Eq. (5) are reached uniformly in $\theta$ and the bundles are analytic if the system is analytic. Indeed, in Refs. 9 and 19 we can find formulas for these rates of growth. In this case, the fact that the limits in Eq. (5) are reached uniformly in $\theta$ implies that $\Lambda^{ \pm}$are the optimal values for $\lambda_{ \pm}$in Eq. (4).

One consequence of the formulas of the theory in Ref. 9 is that the product of both Lyapunov multipliers is the geometric mean of the determinant of $M: \Lambda^{+} \Lambda^{-}=\delta$, where 
$\delta=\exp \int_{\mathrm{T}} \log |\operatorname{det} M(\theta)| d \theta$. (In the examples in this paper, the determinant is constant.) Hence, given one multiplier, $\Lambda^{+}=\Lambda$, we can obtain the other for "free," $\Lambda^{-}=\delta / \Lambda$.

Since the spaces $E_{\theta}^{ \pm}$depend smoothly on $\theta$, the angle between them is bounded away from zero. So, the distance $\Delta$ between the directions $E^{ \pm}$, defined as

$$
\Delta=\min _{\theta \in \mathbb{T}} \angle\left(E_{\theta}^{+}, E_{\theta}^{-}\right),
$$

is strictly positive.

We note that $\Delta$ is closely related to the constant $C$ in the definition of exponential dichotomy in Eq. (4). It is not difficult to obtain upper bounds for the angle in terms of $C$. Heuristically, if the two directions are very close, the rates of growth of vectors on them have to remain close for a long time and, therefore, the constants in Eq. (4) must be large.

The theory of normally hyperbolic manifolds ${ }^{3,20}$ has explicit formulas of the sizes of perturbations for which the persistence of the manifold can be guaranteed. These sizes become very small if the constant $C$ becomes very large or, equivalently, if the norms of the projections $P_{\theta}^{ \pm}$over the invariant directions $E_{\theta}^{ \pm}$become large. Hence, the dichotomy or normal hyperbolicity may become weak even if $\Lambda^{ \pm}$remain safely away from each other but $C$ becomes large.

In this paper we study mechanisms for the disappearance of splittings satisfying Eq. (4). In the literature, one can find several descriptions of the mechanisms in which the Lyapunov multipliers converge to 1 as in a saddle-node bifurcation. $^{21}$ The goal of this paper is to propose a new mechanism based on invariant directions coming closer together while the Lyapunov multipliers remain away from 1 and from each other. We will report several quantitative regularities of this mechanism.

A very recent paper dealing with qualitative features of quasiperiodic systems in which the invariant directions approach each other is Ref. 22.

Our main finding is that, in the situation when the directions come together but the Lyapunov multipliers remain different, there are universal scaling laws. See Assertion 1.

\section{THE DIRECTIONS-MERGING SCENARIOS}

\section{A. Preliminaries}

We consider a system, Eq. (1), depending on a parameter $\varepsilon: f=f_{\varepsilon}$. The algorithms in Ref. 14 allow us to continue a smooth torus $\mathcal{K}_{\varepsilon}$ that satisfies Eq. (4), which is normally hyperbolic.

Hence, it is very natural to use the algorithms in examples and keep on increasing $\varepsilon$ till the continuation runs into trouble. Since we have a well-developed theory that allows us to assess the reliability of the calculation, ${ }^{9,14}$ we can be sure that the observations reported are truly phenomena that happen at the breakdown of hyperbolicity (or exponential dichotomy) and are not artifacts of the calculation.

That is, we expand the torus as a Fourier series and solve Eq. (4) using the Newton method. We perform a continuation of the invariant torus with respect to the parameter $\varepsilon$. We make the numerical experiments in the regions of the param- eter space for which the error estimates are small, say of the order of $10^{-8}$ (in most of the continuation, the errors are smaller than $10^{-15}$ ).

Hence, the theory of Ref. 9 tells us that there is a true invariant torus and that its distance to the computed torus is comparable to the error in solving Eq. (4). Hence, we think that most of the time 10-12 figures of our calculations are correct and, in the worse cases, at least 4 .

Notice that, furthermore, if the torus is attracting we can compute it by using direct iteration of points in phase space. This is used to make additional checks.

The main experiment consists of computing reliably the Lyapunov multipliers $\Lambda_{\varepsilon}^{ \pm}$and the minimum angle between the invariant directions $\Delta_{\varepsilon}$. The main finding of this paper is that both observables fit very well into a scaling relation (see Assertion 1).

The scaling relation predicts that the minimum distance goes to zero as a power of $\left|\varepsilon_{c}-\varepsilon\right|$, where $\varepsilon_{c}$ is a critical value. Moreover, each Lyapunov multiplier goes to a limiting value also as a power of $\left|\varepsilon_{c}-\varepsilon\right|$.

The exponents in the power laws seem to be universal in the sense that small modifications of the system do not alter them. Nevertheless, we have found different universality classes by considering very different systems.

\section{B. The two scenarios studied}

We have studied families in which

(a) The splitting corresponds to two contracting directions (the Lyapunov multipliers do not straddle 1);

(b) The splitting corresponds to one stable and one unstable direction (the Lyapunov multipliers straddle 1). The systems we consider here are area preserving.

In case (a), the attracting-node torus can be continued without difficulty beyond $\varepsilon_{c}$, because it survives after this transition. In case (b) the saddle torus presumably breaks down as a smooth manifold at $\varepsilon=\varepsilon_{c}$ for typical perturbations according to Ref. 4 (unless the perturbation is so special that, e.g., it is zero on the torus).

\section{MAIN QUANTITATIVE RESULTS}

The main result reported in this paper is that in these situations there is some quantitative regularity. Based on numerical evidence, discussed in more detail in Sec. V, we conjecture that:

Assertion 1. For an open set of families $f_{\varepsilon}$, we have: The observables $\Delta$ and $\Lambda^{ \pm}$satisfy the asymptotics

$$
\Delta_{\varepsilon} \sim \alpha\left(\varepsilon_{c}-\varepsilon\right)^{\beta}, \quad \Lambda_{\varepsilon}^{ \pm} \sim \Lambda_{c}^{ \pm}+A^{ \pm}\left(\varepsilon_{c}-\varepsilon\right)^{B}, \quad \varepsilon \lesssim \varepsilon_{c},
$$

where $\alpha, \beta, A^{ \pm}, B$ (and $\Lambda_{c}^{ \pm}$) are fitting parameters.

For systems in case (a), we have: $\beta=1, B=0.5$.

For systems in case (b), we have: $\beta=1, B=1$.

To compare the above powers with some known smooth bifurcations of tori, we note that the saddle-node bifurcation has $\beta=0.5, B=0.5, \Lambda_{c}^{ \pm}=1$, and the transcritical bifurcation has $\beta=1, B=1, \Lambda_{c}^{ \pm}=1$. Since in those the invariant directions remain smooth, one can derive the exponents $\beta, B$ using normal form techniques. In our case, since the invariant di- 
rections become more and more complicated as $\varepsilon$ approaches $\varepsilon_{c}$, there does not seem to be a readily available smooth normal form theory.

In case (a), in which the torus is attracting and survives after the collapse of the fast and slow stable bundles in the critical value $\varepsilon_{c}$, we can also make estimates of the observables after this critical value. Our numerical computations suggest the following:

Assertion 2. For an open set of families $f_{\varepsilon}$ in case (a), we also have the approximations

$$
\Delta_{\varepsilon} \approx 0, \quad \Lambda_{\varepsilon}^{ \pm} \approx \Lambda_{c}^{ \pm}+\bar{A}^{ \pm}\left(\varepsilon-\varepsilon_{c}\right)^{\bar{B}}, \quad \varepsilon \gtrsim \varepsilon_{c},
$$

where $\bar{B}=1$.

While Assertion 1 conjectures asymptotic formulas for the observables, Assertion 2 gives only approximations.

This is quite standard in numerics. When a quantity is not zero, it is easy to ascertain the relative error in its measurement. When the measurement approaches zero, it is possible that a more refined measurement will show some effect.

We also note that the numerics of Assertion 1 can be validated using the well-developed theory of normally hyperbolic systems. Assertion 2 lies in what Ref. 23 refers to as "the dark realm, beyond hyperbolicity, where even the problems are hard to pose clearly," so it is not easy to assess the reliability of the calculations.

Even if we conjecture that $\Delta_{\varepsilon_{c}}=0$, the equality $\Delta_{\varepsilon}=0$ for $\varepsilon \gtrsim \varepsilon_{c}$ would imply that the collapse is produced in an open set of the parameter space. In view of recent mathematical results, ${ }^{24,25}$ this is unlikely to be true. It seems quite possible that there are pockets-perhaps even dense ones!-of parameter values where the values of $\Delta$, even smaller than the precision of the present computation, are nevertheless positive.

In summary, the only thing that Assertion 2 claims is that in the region after collapse, the splitting of the bundles is significantly smaller.

\section{NUMERICAL EVIDENCE}

In this section we summarize the numerical evidence we have for Assertion 1 and Assertion 2. We have verified the results for several other systems similar to the ones presented below, which we hope are representative of different classes of systems.

Of course, it would be interesting to obtain more detailed numerical verifications (more systems, more precision, etc.) and much more interesting still to obtain a justification based on theoretical tools (asymptotic expansions, renormalization group, etc.) or a mathematical proof.

\section{A. Directions merging for an attracting torus}

Our first study is of the rotating Hénon map, ${ }^{26,27}$ a quasiperiodic dissipative map given by

$$
\bar{x}=1+y-a x^{2}+\varepsilon \cos (2 \pi \theta), \quad \bar{y}=b x, \quad \bar{\theta}=\theta+\omega,
$$

where $a$ and $b$ are the parameters of the Hénon map and $\varepsilon$ is the strength of the forcing with frequency $\omega$. In the following, $a=0.68, b=0.1$, and $\omega=\frac{1}{2}(\sqrt{5}-1)$, but we have also con-

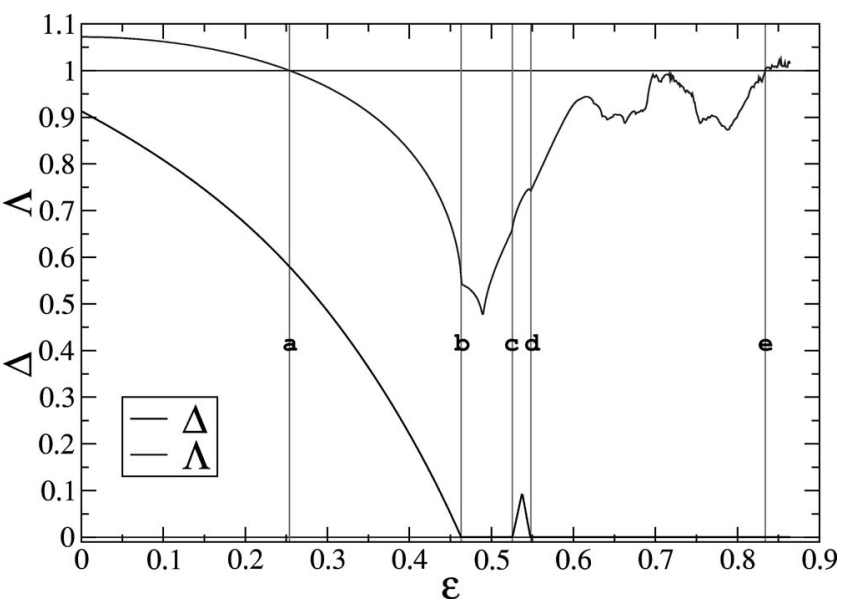

FIG. 1. Observables $\Delta$ and $\Lambda$ as a function of $\varepsilon$.

sidered other families obtained by changing $a, b$, adding extra terms at the map. The results reported in detail below are representative.

For $\varepsilon=0$, the torus $\mathcal{K}(\theta)=\left(x_{0}, b x_{0}, \theta\right)$ with

$$
x_{0}=\frac{1}{2 a}\left(b-1+\sqrt{(b-1)^{2}+4 a}\right)
$$

is invariant and of saddle type. There is also an attracting 2 -periodic torus. We continue with respect to $\varepsilon$, the invariant
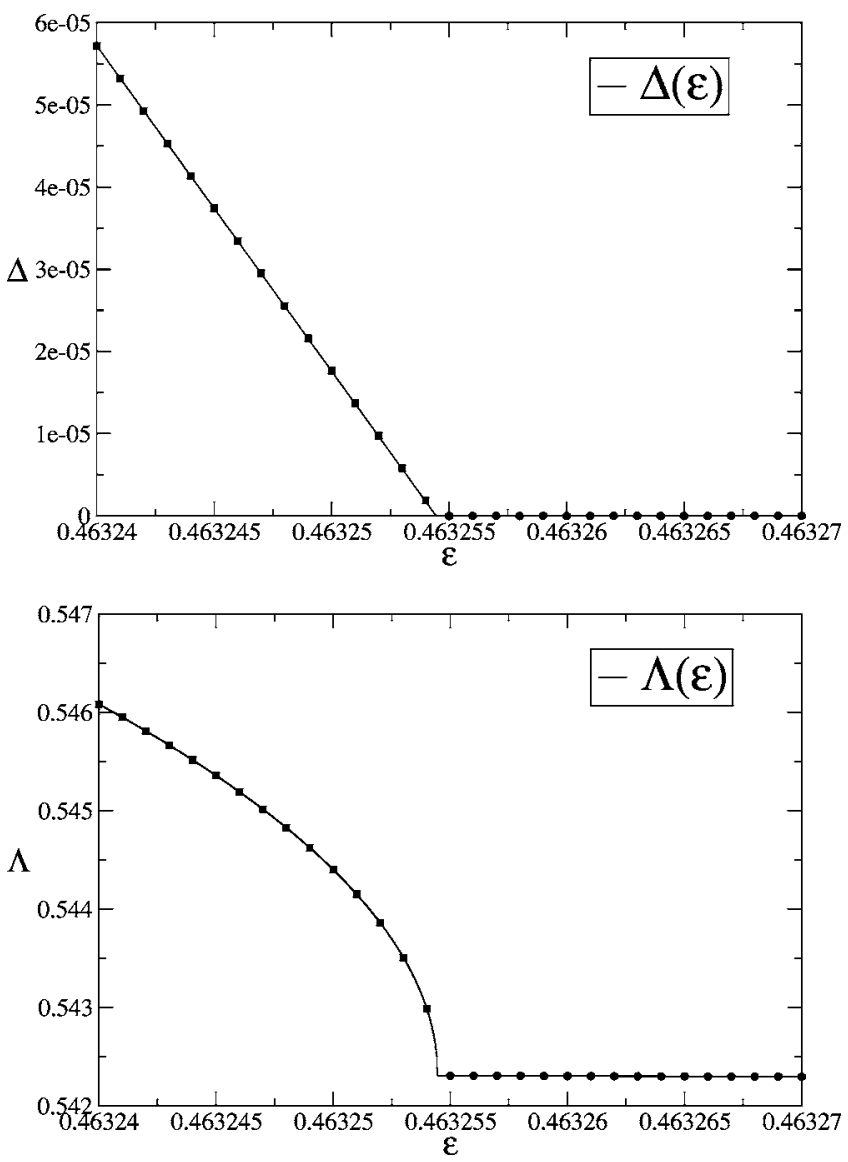

FIG. 2. Fits of $\Delta$ and $\Lambda$ to Eqs. (6) and (7) for the transition $b$, which is produced between $\varepsilon=0.463254$ and $\varepsilon=0.463255$ (see Fig. 3). The fitting parameters are: $\varepsilon_{c}=0.46325447112, \alpha=3.94933, \quad \beta=0.999979$, $\Lambda_{c}=0.5423122, A=1.015, B=0.5020, \bar{A}=-0.7409$, and $\bar{B}=1.00035$. 

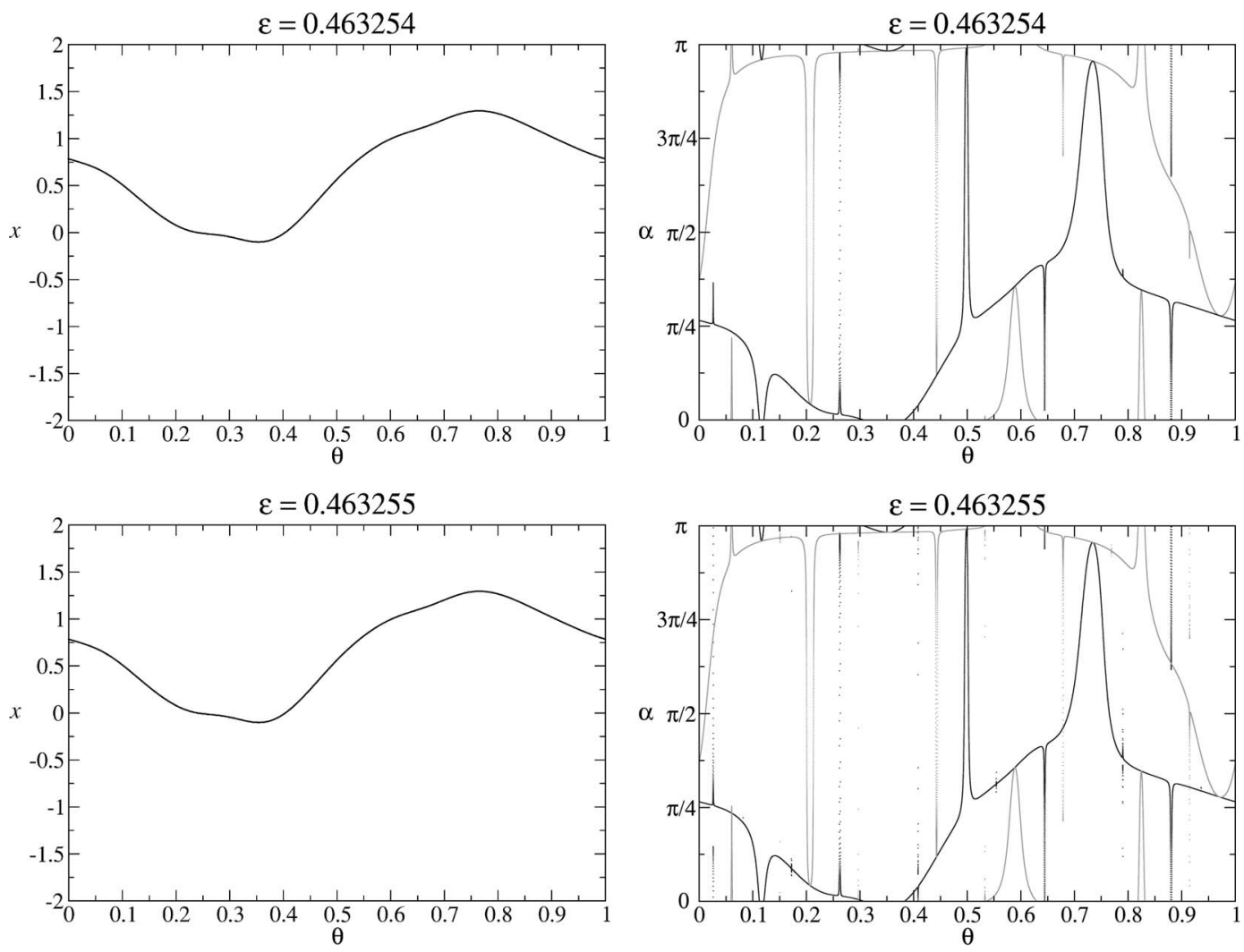

FIG. 3. $x$ projection of an attracting torus and $\alpha$ curves of its slow and fast directions, before and after their collapse. The dramatic change is produced in the invariant objects of the linearization of the dynamics around the torus.

torus, and compute the observables $\Delta$ and $\Lambda=\Lambda^{+}$(notice that $\left.\Lambda^{-}=|b| / \Lambda^{+}\right)$.

The continuation crosses a period-doubling bifurcation, after which the torus is attracting. The computation is very reliable until, say, $\varepsilon=0.62$, at which point the torus starts to wrinkle more wildly and we have to use Fourier expansions of 950 harmonics (although the error estimate is still small, say $\approx 10^{-18}$ ). Since the torus is attracting, we continue beyond this value using direct iteration of the map. We can carry out this continuation even after the invariant object is no longer a smooth manifold.

The results are displayed in Fig. 1, where we observe several transitions labeled with the letters $a, b, c, d, e$. Notice also that throughout the continuation the Lyapunov multipliers remain different (they would be equal only if $\Lambda=\sqrt{|b|}$ $\simeq 0.316$ ).

In $a, \varepsilon_{a} \simeq 0.2540$, there is a standard period "halving" bifurcation, after which the torus is of the attracting-node type. In $b, \varepsilon_{b} \simeq 0.4633$, the slow directions merge with the fast directions. In $c, \varepsilon_{c} \simeq 0.5256$, we have the same phenomenon in reverse and the slow and fast stable directions merge again in $d, \varepsilon_{d} \simeq 0.5475$. In $e, \varepsilon_{e} \simeq 0.8337$ and $\Lambda \simeq 1$, the torus is destroyed.

We focus our discussion on $b$, for which the computations are very reliable. We compute the observables for values of $\varepsilon$ in [0.463 240, 0.463 270], and fit parameters in Eqs. (6) and (7) (see Fig. 2). Notice that $\beta \simeq 1, B \simeq 0.5, \bar{B} \simeq 1$.

As an additional verification, we explore the behavior of the attractor and its invariant directions close to their col- lapse. Each 1D space $E_{\theta}^{ \pm}$is described by an angle $\alpha_{\theta}^{ \pm}$in $[0, \pi]$; hence, the invariant directions $E_{\theta}^{ \pm}$are represented by curves, the angles $\alpha_{\theta}^{ \pm}$depending on $\theta$. We can compute those $\alpha$ curves (the invariant directions as a function of $\theta$ ) using forward and backward iteration of the cocycle $M$ (see Fig. 3).

For $\varepsilon$ close to the computed critical value $\varepsilon_{b}$ there is a very dramatic change in the linearized dynamics around the torus (see Figs. 3 and 4). Notice also the visual similarity to the phenomena mentioned in Refs. 11-13. Similar results happen in $c$ and $d$.

The transition $e$ differs substantially from those mentioned above. Numerically, for $\varepsilon \lesssim \varepsilon_{e}$ it seems that there are no continuous invariant directions even if there are two Lyapunov multipliers (see Fig. 5). At $\varepsilon_{e}$ the maximal Lyapunov multiplier crosses 1 and the torus ceases to be normally hyperbolic; presumably it disappears, leaving behind a more complicated object, which is hard to analyze. The remaining values $\varepsilon>\varepsilon_{e}$ in Fig. 1 would correspond to this object.

Conventionally, this transition around $\varepsilon_{e}$ is called the "fractalization route" 26 (see Ref. 28 for a similar phenomenon in a rotating logistic map), and the invariant object is called strange attractor, nonchaotic if $\varepsilon \leqq \varepsilon_{e}$ or chaotic if $\varepsilon>\varepsilon_{e}$, though its exact nature is not clear. Further numerical experiments add support to the picture mentioned above, i.e., that the torus survives for $\varepsilon \lesssim \varepsilon_{e}$, although it wrinkles quite a bit. $^{29,30}$ (see also Refs. 31 and 32 for a study in a rotating 

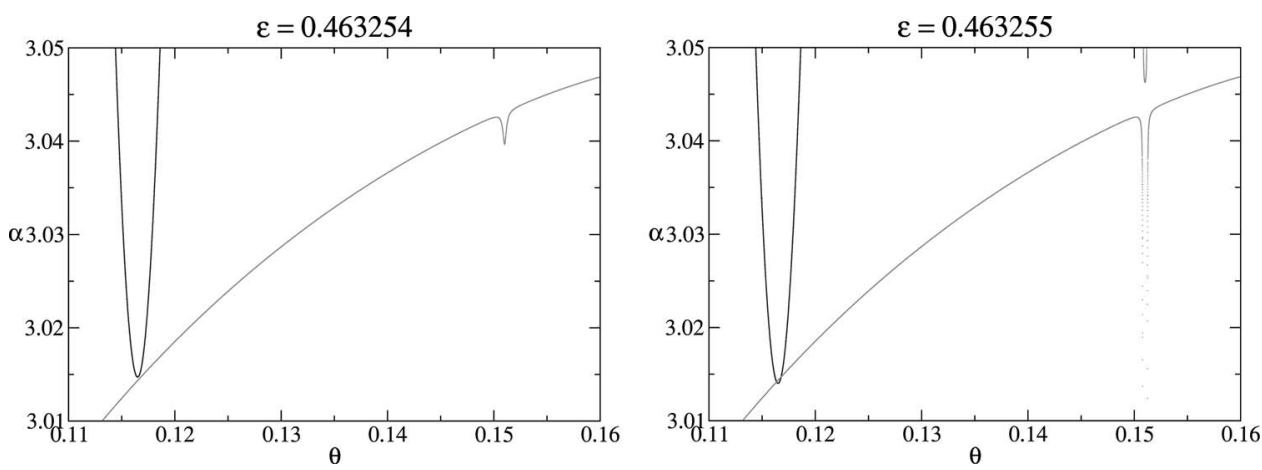

FIG. 4. Zooms of the $\alpha$ curves of Fig. 3 .

logistic map). This transition deserves further study, both from a numerical and a theoretical perspective.

\section{Other external frequencies}

Among many other verifications of universality we have carried out, we display in Fig. 6 an analog of Fig. 2 when $\omega=e / 4$. Note that even if $e / 4$ is numerically close to $(\sqrt{5}$ $-1) / 2$, its detailed number theoretical properties are very different.

\section{B. Directions merging causing breakdown for a saddle torus}

Our second study is of the rotating standard map, ${ }^{33,34}$ a quasiperiodic conservative map given by

$\bar{x}=x+\bar{y}, \quad \bar{y}=y-\frac{K+\varepsilon \cos (2 \pi \theta)}{2 \pi} \sin (2 \pi x), \quad \bar{\theta}=\theta+\omega$,

where $K$ is the parameter of the standard map and $\varepsilon$ leads to a quasiperiodic forcing. In the following, $K=0.2$ and $\omega=\tau-1$, where $\tau$ is the largest root of $p(t)=t^{3}-t^{2}-t-1$.

We continue with respect to $\varepsilon$, an elliptic periodic orbit of period 3 of the standard map. The torus is hyperbolic beyond $\varepsilon \simeq 0.173$. The continuation reaches $\varepsilon=0.377950$, where we use 1200 Fourier harmonics to expand the torus, and the invariance equation Eq. (2) is solved up to an error $\approx 10^{-9} \cdot{ }^{14,35}$ The torus looks rather irregular and about to break and, moreover, the stable and unstable directions are extremely close (see Fig. 7).

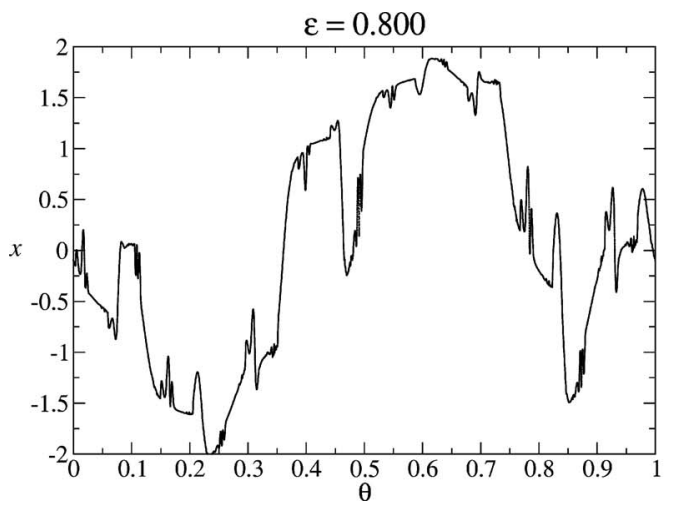

The merging of the invariant directions implies the loss of hyperbolicity and the breakdown of the torus (although the torus can persist as a Cantorus or as a topological manifold). We plan to come back to this problem.

In this case as well we check the scaling laws of the observables $\Delta$ and $\Lambda=\Lambda^{+}$(notice that $\Lambda^{-}=1 / \Lambda^{+}$) by fitting all the parameters (see Fig. 8). Notice that $\beta \simeq 1$ and $B \simeq 1$, and we produce an estimate of the critical value $\varepsilon_{c}$ in which the torus is destroyed.

Compared with the previous example, in this case the torus is destroyed and so is its linearized dynamics, while in the previous one the torus persists after the collapse of the invariant directions.

\section{SOME CONSEQUENCES OF ASSERTION 1}

In the following, we list some mathematical consequences of Assertion 1. The proofs will be published elsewhere since they seem to require a large variety of tools.

We just point out that Assertion 1 lies at the interface of the geometric and functional approaches to normal hyperbolicity.

If Assertion 1 were strictly true, it would have several mathematical consequences, which we list below.

(1) If the attracting and repelling directions coincide at one point, they have to coincide in all the images. Therefore, the collision set, in which the splitting is not defined, has to be dense. Moreover, the invariant directions have to be discontinuous in $\varepsilon=\varepsilon_{c}$. According to Ref. 36, the splitting is

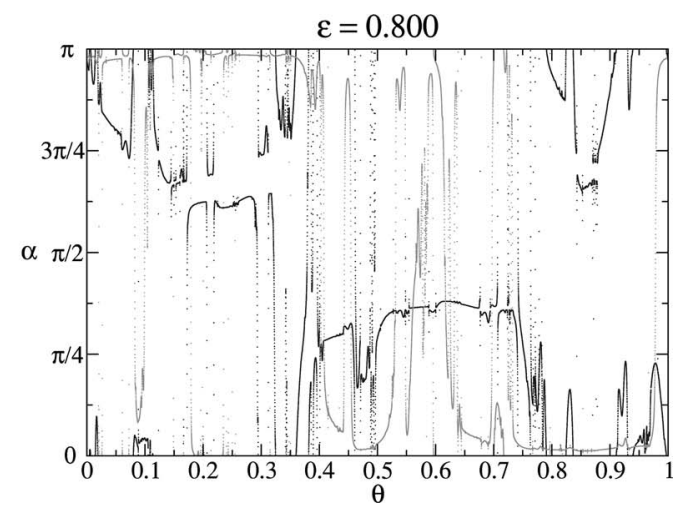

FIG. 5. The $x$ projection of an attracting torus close to breakdown, and $\alpha$ curves of its slow and fast directions. 

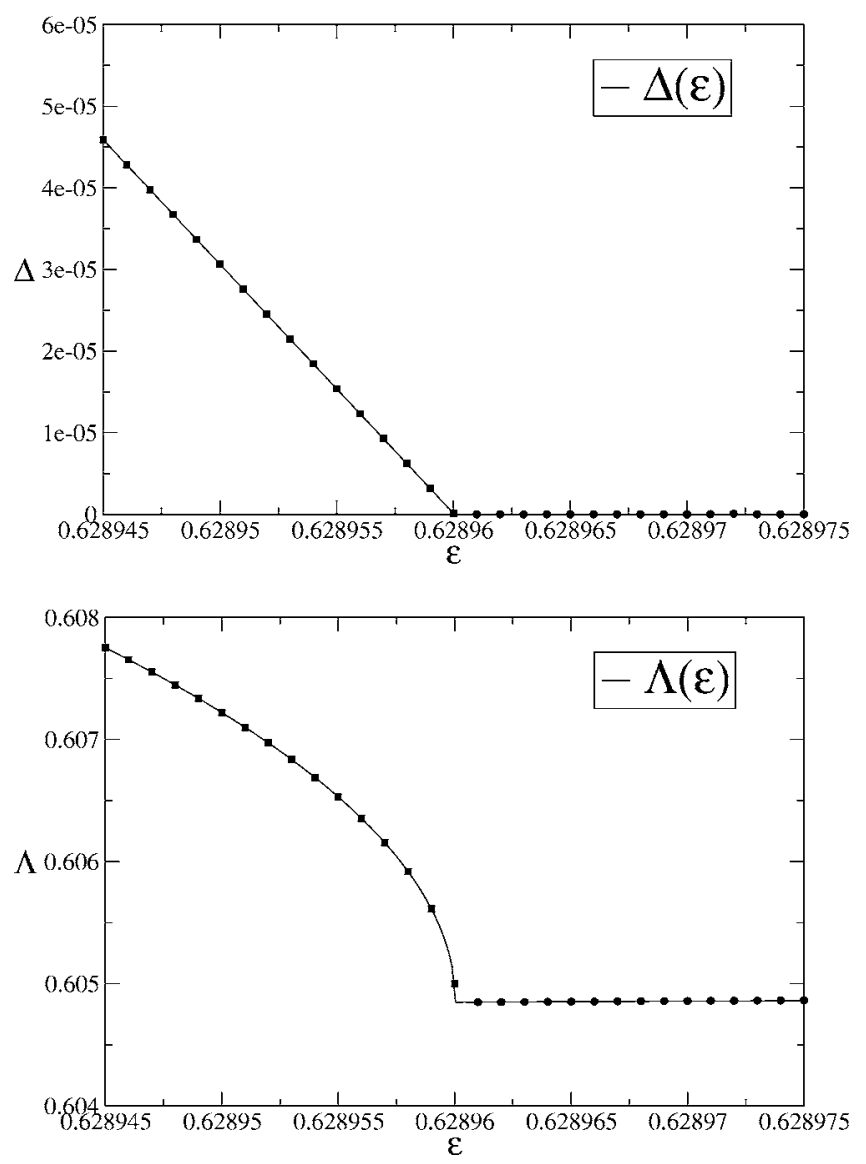

FIG. 6. Fits of $\Delta$ and $\Lambda$ to Eqs. (6) and (7) for a direction-merging bifurcation in an attracting torus of the rotating Hénon map with frequency $\omega=e / 4$, and $a=0.68, b=0.1$. The transition is produced between $\varepsilon=0.628960$ and $\varepsilon=0.628961$. The fitting parameters are: $\varepsilon_{c}=0.62896004195, \quad \alpha=3.0546, \quad \beta=1.000098, \quad \Lambda_{c}=0.60484735$, $A=0.7428, B=0.4993, \bar{A}=0.93$, and $\bar{B}=0.983$.

defined in a set of full measure and the invariant directions are measurable as a function of $\theta$. Hence, the measure of the collision set is zero.

(2) The direction-merging mechanism corresponds to the collision of the $\alpha$ curves that describe the invariant directions. The phenomenon is visually very similar to the formation of strange nonchaotic attractors, which has been described several times in the literature ${ }^{11-13,37}$ (see Figs. 3, 4, and 7).

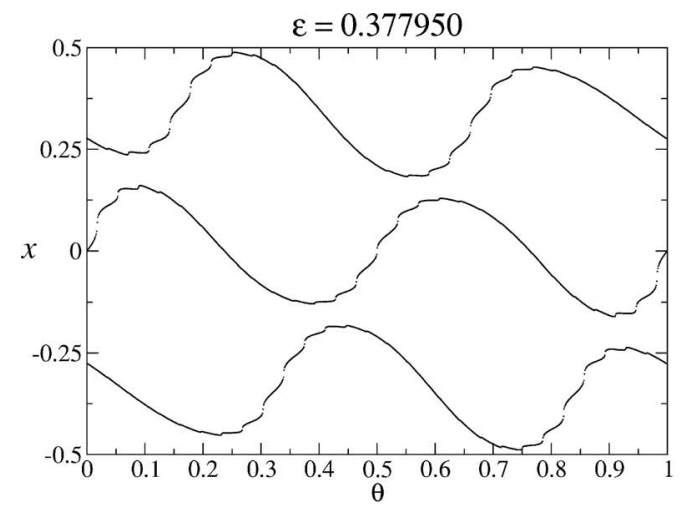

Note that, in spite of their apparent complexity, for $\varepsilon<\varepsilon_{c}$, for the examples considered, the invariant tori and the invariant directions are analytic as a function of $\theta$.

(3) Define the transfer operator $\mathcal{M}$ acting on bounded vector fields $a: T \rightarrow R^{2}$ by

$$
\left(\mathcal{M}_{\bullet}\right)(\theta)=M(\theta-\omega) \propto(\theta-\omega) .
$$

It is known that the spectrum of this operator is related to the existence of invariant splittings as in Eq. (4) ${ }^{38,39}$ Furthermore, using the theory in Refs. 16, 17, and 39, it is possible to show that when the spaces $E_{\theta}^{ \pm}$are one-dimensional, the spectrum is just two circles in the complex plane, centered in the origin. Then, for $\varepsilon<\varepsilon_{c}$ the spectrum is just two circles of radii $\Lambda_{\varepsilon}^{ \pm}$, but for $\varepsilon=\varepsilon_{c}$, the spectrum has to be the full annulus enclosed by these circles. In particular, the spectrum of $\mathcal{M}$ grows discontinuously.

It is well known that the spectrum of a family of bounded linear operators is upper semicontinuous. That is, the spectrum of a limit cannot be smaller than the limit of the spectra but it can be strictly larger. See Ref. 40, IV, Sec. 3.1 for a proof and Ref. 40, IV, Sec. 3.2 for an example of lower semidiscontinuity.

This phenomenon of sudden growth (lower semidiscontinuity) of the spectrum of a family of bounded linear operators is closely related to the fact that the norm of the spectral projections becomes unbounded. The size of the perturbations allowed for the preservation of the gap is smaller if the norm of the spectral projections is larger. In our case, the norm of the spectral projections is precisely $\left(\Delta_{\varepsilon}\right)^{-1}$.

(4) We emphasize that in Assertion 1 we have included the fact that the exponent of the observed power laws is largely independent of the external frequency $\omega$ (see Figs. 2 and 8).

Of course, the exponents of the power laws cannot be completely independent of the frequency. If the frequency is rational, there is no chance that the results are true since the invariant directions solve a finite dimensional equation and, therefore, just experience the standard finite dimensional bifurcations. One can expect that for frequencies given by very Liouville numbers one can produce effects other than the scaling laws mentioned in Assertion 1.

On the other hand, we present numerical evidence that, provided that the frequency is a reasonably irrational num-

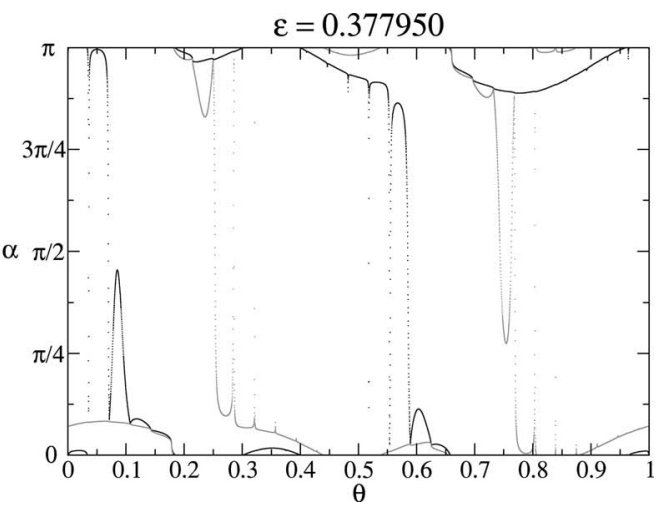

FIG. 7. The $x$ projection of a period 3 torus of the rotating standard map close to breakdown, and the $\alpha$ curves of its unstable and stable directions. 

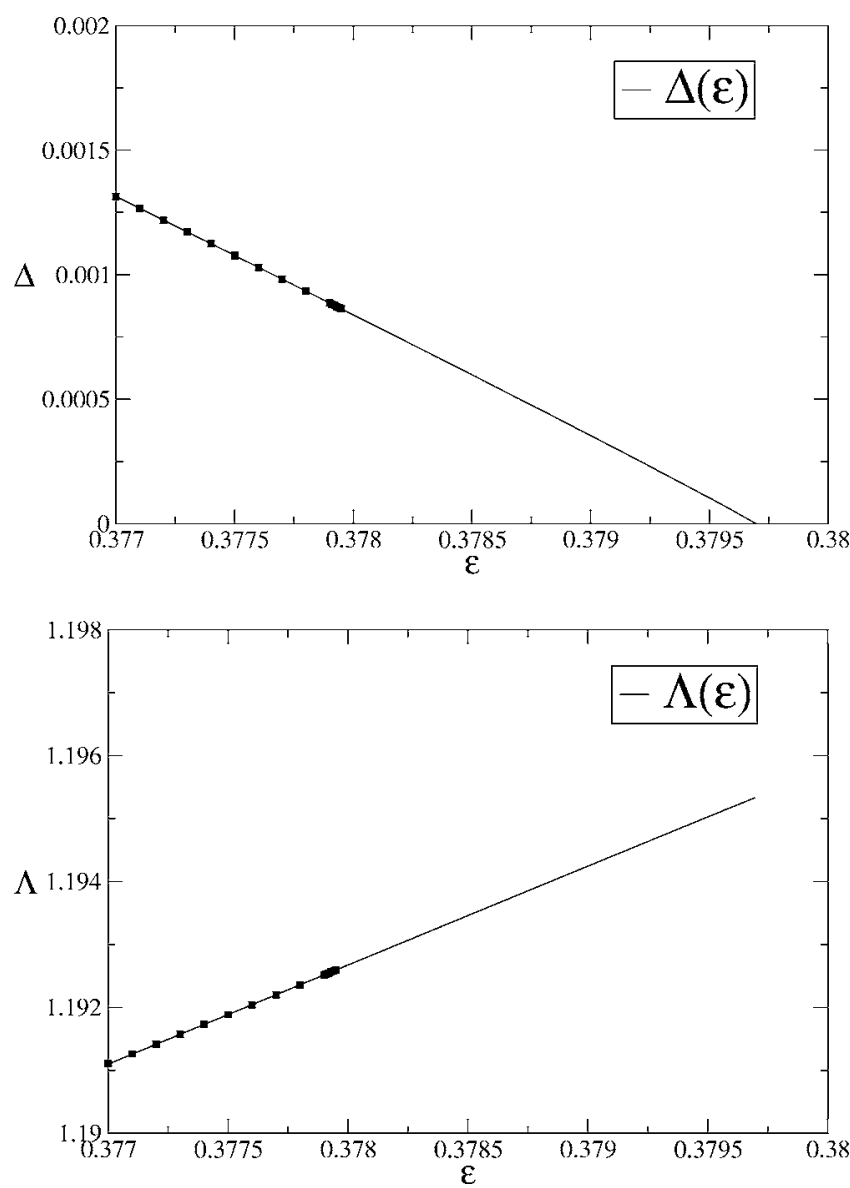

FIG. 8. Fits of $\Delta$ and $\Lambda$ to Eq. (6) near the breakdown of the period 3 torus in the rotating standard map. The fitting parameters are: $\varepsilon_{c}=0.3796965$, $\alpha=0.4063, \beta=0.9693, \Lambda_{c}=1.19533, A=-1.6$, and $B=1.00$.

ber, the results do not depend on the detailed number theoretic properties.

(5) For the systems we are considering, $\Delta>0$ implies that the torus is reducible, that is there exists a linear change of variables (Floquet transformation) that reduces the corresponding cocycle to a constant diagonal matrix ${ }^{16,17}$ (assuming that $\omega$ is Diophantine). Otherwise, $\Delta=0$ implies that the torus is not reducible.

Even if we present numerical evidence for $\Delta \approx 0$ in an open set of the parameter space (see Assertion 2), it is, of course, quite possible that $\Delta>0$ in small pockets of the parameter space that require more precision than that of the numerical computation reported in detail. For instance, one of those pockets is the interval $(0.47186,0.47207)$ of the parameter $\varepsilon$ in the example of Sec. V A (in which we have also verified Assertion 1). A more detailed study is underway. Clearly, feedback between the studies in the recent mathematical literature ${ }^{24,25}$ and the computation will be very important for progress.

\section{CONCLUSIONS}

We have introduced a scenario for the loss of hyperbolicity. We have empirically found that it satisfies scaling relations. There are mathematical consequences of the conjectured scenario.

\section{ACKNOWLEDGMENTS}

A.H. is supported by the MCyT/FEDER Grant BFM2003-07251-C02-01 and the CIRIT Grant 2001SGR-70. R.L. is supported by NSF grants and ICREA.

${ }^{1}$ R. J. Sacker, Comm. Pure Appl. Math. 18, 717 (1965).

${ }^{2}$ M. W. Hirsch and C. C. Pugh, Bull. Amer. Math. Soc. 75, 149 (1969).

${ }^{3}$ N. Fenichel, Indiana Univ. Math. J. 21, 193 (1971/1972).

${ }^{4}$ R. Mañé, Trans. Amer. Math. Soc. 246, 261 (1978).

${ }^{5}$ J. Palis, Astérisque (Societé Mathematique de France, Paris, France, 2000), pp. xiii-xiv, 335-347. Géométrie Complexe et Systèmes Dynamiques (Societé Mathematique de France, Paris, France, 1995).

${ }^{6} \mathrm{~K}$. Kaneko, Collapse of Tori and Genesis of Chaos in Dissipative Systems (World Scientific Publishing Co., Singapore, 1986).

${ }^{7}$ C. Bonatti, L. Diaz, and M. Viana, Dynamics Beyond Uniform Hyperbolicity: A Global Geometric And Probabilistic Perspective (SpringerVerlag, Berlin, 2004).

${ }^{8}$ C. Chicone and W. Liu, SIAM J. Math. Anal. 31, 386 (1999-2000).

${ }^{9}$ À. Haro and R. de la Llave, J. Differential Equations (to appear).

${ }^{10}$ C. Grebogi, E. Ott, S. Pelikan, and J. A. Yorke, Phys. D 13, 261 (1984).

${ }^{11}$ P. Glendinning, U. Feudel, A. S. Pikovsky, and J. Stark, Phys. D 140, 227 (2000).

${ }^{12}$ H. Osinga, J. Wiersig, P. Glendinning, and U. Feudel, Int. J. Bifurcation Chaos Appl. Sci. Eng. 11, 3085 (2001).

${ }^{13}$ A. Prasad, S. S. Negi, and R. Ramaswamy, Int. J. Bifurcation Chaos Appl. Sci. Eng. 11, 291 (2001).

${ }^{14} \grave{A}$. Haro and R. de la Llave, mp_arc 04-350 (2004).

${ }^{15}$ R. Sacker and G. Sell, J. Differential Equations 15, 429 (1974).

${ }^{16}$ R. A. Johnson, J. Differential Equations 35, 366 (1980).

${ }^{17}$ R. A. Johnson and G. R. Sell, J. Differential Equations 41, 262 (1981).

${ }^{18}$ C. Falcolini and R. de la Llave, J. Stat. Phys. 67, 609 (1992).

${ }^{19}$ C. Chicone and R. C. Swanson, J. Differential Equations 40, 155 (1981).

${ }^{20}$ M. Hirsch, C. Pugh, and M. Shub, Invariant Manifolds, Lecture Notes in Mathematics, Vol. 583(Springer-Verlag, Berlin, 1977).

${ }^{21}$ A. Chenciner and G. Iooss, Arch. Rational Mech. Anal. 69, 109 (1979).

${ }^{22}$ A. Y. Jalnine and A. Osbaldestin, Phys. Rev. E 71, 016206 (2005).

${ }^{23}$ S. Smale, Phys. D 51, 267 (1991).

${ }^{24}$ A. Avila and R. Krikorian, Preprint (2003).

${ }^{25}$ A. Krikorian, Preprint (2004).

${ }^{26}$ O. Sosnovtseva, U. Feudel, J. Kurths, and A. Pikovsky, Phys. Lett. A 218 255 (1996).

${ }^{27}$ S.-Y. Kim and W. Lim, J. Phys. A 37, 6477 (2004).

${ }^{28}$ T. Nishikawa and K. Kaneko, Phys. Rev. E 54, 6614 (1996).

${ }^{29}$ A. Haro and R. de la Llave, SIAM Conference on Applications of Dynamical Systems (Snowbird, UT, 22-26 May 2005).

${ }^{30}$ À. Haro and C. Simó (2005), mp_arc \# 05-429.

${ }^{31}$ H. Broer, C. Simó, and R. Vitolo (2005), mp_arc \#05-107.

${ }^{32}$ A. Jorba and J. C. Tatjer, preprint (2005), mparc \#05-429.

${ }^{33}$ R. Artuso, G. Casati, and D. L. Shepelyansky, Europhys. Lett. 15, 381 (1991).

${ }^{34}$ S. Tompaidis, Experiment. Math. 5, 211 (1996).

${ }^{35}$ À. Haro and R. de la Llave, mp_arc 05-246 (2005).

${ }^{36}$ V. I. Oseledec, Trudy Moskov. Mat. Obšč. 19, 179 (1968).

${ }^{37}$ G. Keller, Fund. Math. 151, 139 (1996), ISSN 0016-2736.

${ }^{38}$ J. N. Mather, Nederl. Akad. Wetensch. Proc. Ser. A $71=$ Indag. Math. 30, 479 (1968).

${ }^{39} \grave{A}$. Haro and R. de la Llave, Preprint (2003).

${ }^{40}$ T. Kato, Perturbation Theory for Linear Operators, 2nd ed. (SpringerVerlag, Berlin, 1976), Grundlehren der Mathematischen Wissenschaften, Band 132 . 